PROCEEDINGS OF THE

AMERICAN MATHEMATICAL SOCIETY

Volume 124, Number 11, November 1996

\title{
MINIMAL UPPER BOUNDS OF COMMUTING OPERATORS
}

\author{
CHARLES AKEMANN AND NIK WEAVER
}

(Communicated by Palle E. T. Jorgensen)

\begin{abstract}
Let $\left(x_{i}\right)$ be a finite collection of commuting self-adjoint elements of a von Neumann algebra $\mathcal{M}$. Then within the (abelian) $\mathrm{C}^{*}$-algebra they generate, these elements have a least upper bound $x$. We show that within $\mathcal{M}, x$ is a minimal upper bound in the sense that if $y$ is any self-adjoint element such that $x_{i} \leq y \leq x$ for all $i$, then $y=x$. The corresponding assertion for infinite collections $\left(x_{i}\right)$ is shown to be false in general, although it does hold in any finite von Neumann algebra.

We use this sort of result to show that if $\mathcal{N} \subset \mathcal{M}$ are von Neumann algebras, $\Phi: \mathcal{M} \rightarrow \mathcal{N}$ is a faithful conditional expectation, and $x \in \mathcal{M}$ is positive, then $\Phi\left(x^{n}\right)^{1 / n}$ converges in the strong operator topology to the "spectral order majorant" of $x$ in $\mathcal{N}$.
\end{abstract}

This paper arose from an investigation of the sequence $\left(x^{n}+y^{n}\right)^{1 / n}$, where $x$ and $y$ are positive semidefinite operators on a Hilbert space. If $x$ and $y$ commute, i.e. $x, y \in C(X)$ (the continuous complex-valued functions on a compact Hausdorff space $X)$, then this sequence decreases to their least upper bound in $C(X)$. We found that in general it converges in the strong operator topology, and the limit operator is the least upper bound of $x$ and $y$ relative to a nonstandard ("spectral") order on $B(H)$ (the algebra of bounded linear operators on the Hilbert space $H$ ). After proving this result we learned that it had already been proved in [7] (see also [1] and [14]). However, we also became acquainted with closely related work of Alan Lambert which suggested a generalization in terms of conditional expectations. Namely, if $\mathcal{N} \subset \mathcal{M}$ are von Neumann algebras, $\Phi: \mathcal{M} \rightarrow \mathcal{N}$ is a faithful conditional expectation, and $x \in \mathcal{M}$ is positive, then $\Phi\left(x^{n}\right)^{1 / n}$ converges to the least element of $\mathcal{N}$ which is greater than $x$, relative to the spectral order on $\mathcal{M}$. This result is discussed in section II. We thank Alan Lambert for calling our attention to the similarity between his and our work and suggesting that there might be a relation.

In our investigation the following question arose. Let $\left(x_{i}\right)$ be a collection of commuting self-adjoint operators and let $x$ be their least upper bound in the (commutative) von Neumann algebra they generate. Then is $x$ a minimal upper bound for $\left(x_{i}\right)$ within $B(H)$ ? We found that this is not always so, although it is true if the collection $\left(x_{i}\right)$ is finite; also, within finite von Neumann algebras, it is true for infinite collections (section I). In addition, Kato [7] has shown that any positive operator $x$ is a minimal upper bound for the operators $a E_{x}(a, \infty)$, where $E_{x}$ is the

Received by the editors June 5, 1995.

1991 Mathematics Subject Classification. Primary 47C15, 46L10.

This research was partially supported by NSF grant DMS-9424370.

(C)1996 American Mathematical Society 
spectral measure of $x$ and $a$ ranges over $\mathbf{R}$ (Theorem 6); this is the crucial result needed to show convergence of $\Phi\left(x^{n}\right)^{1 / n}$.

\section{MinimaL UPPER BOUNDS}

Let $H$ be a (complex) Hilbert space and $B(H)$ the algebra of bounded operators on $H$. According to a well-known result of Kadison [5], two self-adjoint operators $x, y \in B(H)$ have a least upper bound (or "join") in $B(H)$ if and only if they are comparable, i.e. $x \leq y$ or $x \geq y$. A related result of Sherman [11] states that the self-adjoint elements of a $\mathrm{C}^{*}$-algebra $\mathcal{A}$ form a lattice if and only if $\mathcal{A}$ is abelian.

On the other hand, any bounded collection of commuting self-adjoint operators $\left(x_{i}\right)$ does have a join $x$ within the (abelian) von Neumann algebra it generates. We call $x$ the commutative join of the $x_{i}$. Note that $x$ remains a join of $\left(x_{i}\right)$ within any commutative algebra containing $\left(x_{i}\right)$; this is clear by a functional calculus argument if the collection is finite [5, Lemma 10] and then follows for any collection by taking the limit of a net of finite joins.

By the preceding results, we cannot expect $x$ to be a join within larger algebras in general (e.g., in $B(H)$ ); but we can still ask whether $x$ is at least a minimal upper bound for the collection $\left(x_{i}\right)$, within a larger algebra. In this connection the following result seems to be useful. Recall that if $\mathcal{M}$ and $\mathcal{N}$ are von Neumann algebras and $\mathcal{N} \subset \mathcal{M}$, then a conditional expectation from $\mathcal{M}$ onto $\mathcal{N}$ is a positive linear mapping $\Phi: \mathcal{M} \rightarrow \mathcal{N}$ such that $\Phi(I)=I$ and $\Phi(x y z)=x \Phi(y) z$ for all $x, z \in \mathcal{N}$ and $y \in \mathcal{M}$ (see [6, p. 834]). Equivalently, $\Phi$ is a projection of norm 1 from $\mathcal{M}$ onto $\mathcal{N}$ [13]. It is called faithful if $x>0$ implies $\Phi(x)>0$.

Proposition 1. Let $\mathcal{M}$ and $\mathcal{N}$ be von Neumann algebras, $\mathcal{N} \subset \mathcal{M}$, and suppose there exists a faithful conditional expectation $\Phi: \mathcal{M} \rightarrow \mathcal{N}$. Let $\left(x_{i}\right) \subset \mathcal{N}$ be a bounded collection of self-adjoint elements and suppose $x \in \mathcal{N}$ is a minimal upper bound of $\left(x_{i}\right)$ within $\mathcal{N}$. Then $x$ remains a minimal upper bound for $\left(x_{i}\right)$ within $\mathcal{M}$.

Proof. Let $y \in \mathcal{M}$ and suppose $x_{i} \leq y \leq x$ for all $i$. Since $\Phi$ is positive and idempotent, it follows that

$$
x_{i}=\Phi\left(x_{i}\right) \leq \Phi(y) \leq \Phi(x)=x
$$

for all $i$. Thus $\Phi(y)=x$, i.e. $\Phi(x-y)=0$, by the minimality of $x$ within $\mathcal{N}$. But then $y<x$, i.e. $x-y>0$, would contradict the faithfulness of $\Phi$. We conclude that $y=x$, which shows that $x$ is a minimal upper bound within $\mathcal{M}$.

The following three corollaries give conditions under which the commutative join of $\left(x_{i}\right)$ is a minimal upper bound, within a von Neumann algebra $\mathcal{M}$. This conclusion can be drawn if $\mathcal{M}$ is finite, or if the collection $\left(x_{i}\right)$ is finite, or if $\left(x_{i}\right) \subset l^{\infty}$.

Corollary 2. Let $\mathcal{M}$ be a finite von Neumann algebra and let $\left(x_{i}\right)$ be a bounded collection of commuting self-adjoint elements of $\mathcal{M}$. Then the commutative join of the $x_{i}$ is a minimal upper bound for the $x_{i}$ within $\mathcal{M}$.

Proof. By Zorn's lemma we can find an orthogonal family $\left(p_{k}\right)$ of central projections in $\mathcal{M}$ such that $\sum p_{k}=1$, together with tracial states $\left(f_{k}\right)$ on $\mathcal{M}$ such that $f_{k}\left(p_{k}\right)=$ 1 for all $k$ and such that each $f_{k}$ is faithful on $p_{k} \mathcal{M}$. Let $\mathcal{N}$ be the von Neumann algebra generated by $\left(p_{k}\right)$ and $\left(x_{i}\right)$. Then by ([4, Théoréme 8$]$; or [12, p. 332]), there 
exists a faithful conditional expectation $\Phi_{k}: p_{k} \mathcal{M} \rightarrow p_{k} \mathcal{N}$ for all $k$, and summing gives us a faithful conditional expectation $\Phi: \mathcal{M} \rightarrow \mathcal{N}$.

Let $x$ be the commutative join of the $x_{i}$. Since $\mathcal{N}$ is commutative, $x$ is the least upper bound of the $x_{i}$ in $\mathcal{N}$, so it is certainly a minimal upper bound within $\mathcal{N}$. Proposition 1 now implies that $x$ is a minimal upper bound within $\mathcal{M}$ as well.

Corollary 3. Let $\mathcal{M}$ be a von Neumann algebra and let $\left(x_{i}\right)$ be a finite collection of commuting self-adjoint elements of $\mathcal{M}$. Then the commutative join of the $x_{i}$ is a minimal upper bound for the $x_{i}$ within $\mathcal{M}$.

Proof. Without loss of generality suppose $\mathcal{M}=B(H)$. Let $x$ be the commutative join of the $x_{i}$. We begin by claiming that there is a collection of projections $\left(p_{i}\right)$ which commute with the $x_{i}$ and satisfy $\sum p_{i}=1$ and $p_{i} x_{i}=p_{i} x$ for all $i$. These can be constructed inductively, setting $p_{i}^{\prime}=$ the projection onto $\operatorname{ker}\left(x-x_{i}\right)$ and

$$
p_{i}=\left(1-\sum_{1}^{i-1} p_{j}\right) p_{i}^{\prime} .
$$

It is easy to see that $\sum p_{i}=1$ by looking at the (abelian) $\mathrm{C}^{*}$-algebra generated by the $x_{i}$ and the $p_{i}$.

Now let $\mathcal{N}$ be the commutant within $\mathcal{M}$ of the collection $\left(p_{i}\right)$. Then the map $\Phi: y \mapsto \sum p_{i} y p_{i}$ is a conditional expectation from $\mathcal{M}$ onto $\mathcal{N}$, and we claim it is also faithful. To see this suppose $y \geq 0$ and $\Phi(y)=0$. Then for each $i$ we have

$$
p_{i} y p_{i}=\left(y^{1 / 2} p_{i}\right)^{*}\left(y^{1 / 2} p_{i}\right)=0,
$$

hence $y^{1 / 2} p_{i}=0$, hence $y p_{i}=0$. Summing over $i$ then shows that $y=0$. Thus $\Phi$ is faithful, as claimed.

To complete the proof we must show that $x$ is a minimal upper bound for $\left(x_{i}\right)$ within $\mathcal{N}$. In fact, $x$ is the least upper bound within $\mathcal{N}$. For suppose $y \in \mathcal{N}$ and $y \geq x_{i}$ for all $i$. Then

$$
p_{i} y \geq p_{i} x_{i}=p_{i} x
$$

for all $i$, so that

$$
y=\sum p_{i} y \geq \sum p_{i} x=x
$$

Thus within $\mathcal{N}, x$ is the join of the $\left(x_{i}\right)$, so by Proposition 1 it remains minimal within $\mathcal{M}$.

Corollary 4. Let $\mathcal{M}$ be a von Neumann algebra and let $\left(x_{i}\right) \subset \mathcal{M}$ be a bounded collection of commuting self-adjoint elements. Suppose that the von Neumann algebra generated by $\left(x_{i}\right)$ is isomorphic to $l^{\infty}(S)$ for some index set $S$. Then the commutative join of the $x_{i}$ is a minimal upper bound for the $x_{i}$ within $\mathcal{M}$.

Proof. Identify $l^{\infty}(S)$ with the von Neumann algebra generated by $\left(x_{i}\right)$ and let $\mathcal{N}$ be the commutant of $l^{\infty}(S)$ within $\mathcal{M}$. Let $\left(p_{i}\right)$ be the set of minimal projections in $l^{\infty}(S)$. Then we have a conditional expectation $\Phi: \mathcal{M} \rightarrow \mathcal{N}$ defined by $\Phi(y)=$ $\sum p_{i} y p_{i}$, and it is faithful by the same argument as used in Corollary 3 . Also, $x$ is a minimal upper bound for $\left(x_{i}\right)$ (in fact, a join) within $\mathcal{N}$, again as in Corollary 3 ; so Proposition 1 implies that $x$ is minimal in $\mathcal{M}$. 
In light of the preceding results it seems reasonable to conjecture that any commutative join is always a minimal upper bound. However, we will now show that this is not the case. First observe that by negating every operator, the question about joins is equivalent to a similar question about meets (greatest lower bounds); this is the version that we will falsify. For $n \in \mathbf{N}$ and $1 \leq k \leq n$ define $f_{n, k}:[0,1] \rightarrow \mathbf{R}$ by

$$
f_{n, k}(t)= \begin{cases}2 / n & \text { if } \frac{k-1}{n} \leq t \leq \frac{k}{n} \\ 2 & \text { otherwise }\end{cases}
$$

Then let $M_{n, k}$ be the operator on $L^{2}[0,1]$ given by multiplication by $f_{n, k}$. It is easy to see that the operators $M_{n, k}$ all commute, and their commutative meet - their greatest lower bound within $L^{\infty}[0,1]$ - is 0 .

Let 1 denote the function in $L^{2}[0,1]$ which is constantly one, and let $P$ be the orthogonal projection onto 1 ; thus for any $g \in L^{2}[0,1], P g=\langle g, 1\rangle 1$ is the function which takes the constant value

$$
\langle g, 1\rangle=\int_{0}^{1} g(t) d t
$$

It is clear that $P$ is strictly positive, and we will now show that $P \leq M_{n, k}$ for all $n, k$.

To see this, let $g \in L^{2}[0,1]$ and note that $\int_{0}^{1}\left(f_{n, k}(t)\right)^{-1} d t \leq 1$. So

$$
\begin{aligned}
\langle P g, g\rangle & =\left|\int_{0}^{1} g(t) d t\right|^{2} \\
& \leq\left(\int_{0}^{1}\left(f_{n, k}(t)\right)^{1 / 2}|g(t)|\left(f_{n, k}(t)\right)^{-1 / 2} d t\right)^{2} \\
& \leq\left(\int_{0}^{1} f_{n, k}(t)|g(t)|^{2} d t\right)\left(\int_{0}^{1}\left(f_{n, k}(t)\right)^{-1} d t\right) \\
& \leq \int_{0}^{1} f_{n, k}(t)|g(t)|^{2} d t \\
& =\left\langle M_{n, k} g, g\right\rangle .
\end{aligned}
$$

(The crucial middle inequality is the Cauchy-Schwarz inequality.) Thus, we conclude that $0<P \leq M_{n, k}$ for all $n, k$ even though 0 is the commutative meet of the $M_{n, k}$. This completes the example.

There is a simple formula for the commutative join of two operators:

$$
x_{1} \vee x_{2}=\left(x_{1}+x_{2}+\left|x_{1}-x_{2}\right|\right) / 2 .
$$

This formula still makes sense if $x_{1}$ and $x_{2}$ fail to commute, which suggests the following result.

Corollary 5. Let $x_{1}$ and $x_{2}$ be two self-adjoint elements of a von Neumann algebra. Then $x=\left(x_{1}+x_{2}+\left|x_{1}-x_{2}\right|\right) / 2$ is a minimal upper bound for $x_{1}$ and $x_{2}$.

Proof. Without loss of generality we may assume that the ambient von Neumann algebra is $B(H)$. Note first that

$$
2 x_{1}=x_{1}+x_{2}+\left(x_{1}-x_{2}\right) \leq x_{1}+x_{2}+\left|x_{1}-x_{2}\right|,
$$


hence $x_{1} \leq x$. Similarly $x_{2} \leq x$, so that $x$ is an upper bound for $x_{1}$ and $x_{2}$. Conversely let $y$ be self-adjoint and suppose $x_{1}, x_{2} \leq y \leq x$. Then some easy algebra shows that

$$
x_{1}-x_{2}, x_{2}-x_{1} \leq 2 y-x_{1}-x_{2} \leq\left|x_{1}-x_{2}\right| .
$$

Now the operators $x_{1}-x_{2}$ and $x_{2}-x_{1}$ commute and their commutative join is $\left|x_{1}-x_{2}\right|$, so Corollary 3 implies that

$$
2 y-x_{1}-x_{2}=\left|x_{1}-x_{2}\right| .
$$

From this it follows that $y=x$.

Finally, let us mention that the join of a set of projections (i.e. the projection onto the closed linear span of their ranges) is a minimal upper bound within any ambient von Neumann algebra. By complementation (i.e., replacing $p$ by $1-p$ ), this is implied by the following result of Kadison ([5, Lemma 2]): let $\left(p_{i}\right)$ be a set of projections and let $p$ be their meet (i.e. the projection onto the intersection of the ranges); then among all positive operators, $p$ is a greatest lower bound.

(Kadison states this result for two projections, but the proof works for arbitrary collections. A von Neumann algebra argument goes as follows. Let $\left(p_{i}\right)$ be a set of projections in a von Neumann algebra $\mathcal{M}$ and let $q$ be their join in the lattice of projections, i.e. the projection onto the closed linear span of the ranges. Suppose $x \in \mathcal{M}$ satisfies $p_{i} \leq x \leq q$ for all $i$. Then $x-p_{i}$ is positive and dominated by $q-p_{i}$, hence $x-p_{i}$ commutes with $p_{i}$, hence $x$ commutes with $p_{i}$. Now since $p_{i} \leq x$ and the two commute, it follows that $p_{i} \leq E_{x}\{1\}$, and as this is true for all $i$ we conclude that $q \leq E_{x}\{1\}$, hence $q \leq x$.)

Our final result along the lines of this section was given in ([7, Lemma $]$ ). (We thank Khristo Boyadzhiev for bringing this to our attention.) For $I \subset \mathbf{R}$ and $x$ a self-adjoint element of a von Neumann algebra, let $E_{x}(I)$ denote the $x$-spectral projection of $I$. The result states that any positive $x$ is a minimal upper bound for the collection of operators $\left\{a E_{x}(a, \infty): a \in \mathbf{R}\right\}$. As these operators commute and their commutative join is $x$, this follows from Corollary 2 if the von Neumann algebra is finite. Also, it follows from Corollary 4 if $x$ has a discrete spectrum. However in general the proof is a bit more involved.

Theorem 6 (Kato). Let $x$ and $y$ be positive elements of a von Neumann algebra $\mathcal{M}$ and suppose $a E_{x}(a, \infty) \leq y \leq x$ for all $a \in \mathbf{R}$. Then $y=x$.

\section{Convergence of $\Phi\left(x^{n}\right)^{1 / n}$}

In [9] Olson introduced a new partial order with respect to which the self-adjoint elements of any von Neumann algebra form a conditionally complete lattice - i.e. every bounded set has a least upper bound and greatest lower bound. (This order seems to have been rediscovered several times. It was independently introduced in $[2,3]$, and [10], and perhaps elsewhere.) This "spectral order" is defined by $x \preceq y$ if and only if $E_{x}(a, \infty) \leq E_{y}(a, \infty)$ for all $a \in \mathbf{R}$. Indeed, it is easy to see that any bounded collection of self-adjoint elements $\left\{x_{i}\right\}$ has a join with respect to this order; it is that self-adjoint element $y$ such that

$$
E_{y}(a, \infty)=\bigvee E_{x_{i}}(a, \infty)
$$

for all $a \in \mathbf{R}$. ( $\bigvee$ denotes the join of projections, i.e. closed linear span.) Olson also showed that $x \preceq y$ if and only if $x^{n} \leq y^{n}$ for all $n \in \mathbf{N}$. 
The spectral order join behaves as one would expect in familiar cases. For instance, if $x$ and $y$ commute then it is just the commutative join of $x$ and $y$ discussed in section I. Or, if $x$ and $y$ are (perhaps noncommuting) projections, then their join in the spectral order is just their join as projections.

(In light of section I, it is worth noting that the spectral order join is not always a minimal upper bound relative to the usual order. For instance, consider the $2 \times 2$ matrices

$$
\left(\begin{array}{ll}
8 & 0 \\
0 & 0
\end{array}\right),\left(\begin{array}{ll}
8 & 8 \\
8 & 8
\end{array}\right)<\left(\begin{array}{cc}
11 & 5 \\
5 & 11
\end{array}\right)<\left(\begin{array}{cc}
12 & 4 \\
4 & 12
\end{array}\right)
$$

The rightmost matrix is the spectral order join of the two on the left, as one can see by checking eigenspaces.)

Now let $\mathcal{N} \subset \mathcal{M}$ be von Neumann algebras, $\Phi: \mathcal{M} \rightarrow \mathcal{N}$ a faithful conditional expectation, and $x \in \mathcal{M}$ a positive element. (We retain this notation in Lemmas 7 and 8.) The collection

$$
\left\{y=y^{*} \in \mathcal{N}: x \preceq y\right\}
$$

is bounded below and hence has a greatest lower bound with respect to the spectral order in $\mathcal{N}$. Thus, there exists a least element $x_{+}$of $\mathcal{N}$ among all those which dominate $x$ in spectral order. Equivalently, $x_{+}$can be defined by specifying its spectral projections, namely $E_{x_{+}}(a, \infty)$ is the least projection in $\mathcal{N}$ which contains $E_{x}(a, \infty)$. We want to prove that the sequence $\Phi\left(x^{n}\right)^{1 / n}$ converges in the strong operator topology to $x_{+}$.

In the case of abelian $\mathcal{M}$ and $\mathcal{N}$ this result is due to Lambert [8]. Here the spectral order agrees with the usual order, so that $x_{+}$is the majorant of $x$ in $\mathcal{N}$ in the usual sense; thus Lambert's result gives a characterization of this majorant in terms of the moments of $x$. Our result (Theorem 9) can be seen as a quantum probabilistic version of this fact.

Lemma 7. Let $y \in \mathcal{M}, y \geq 0$, and let $p$ be its range projection. Then $y^{1 / n} \rightarrow p$ in the strong operator topology.

Proof. Let $\mathcal{M}$ act normally on a Hilbert space $H$. First observe that the set of vectors $\xi$ such that $E_{y}(0, \epsilon) \xi=0$ for some $\epsilon>0$ is dense in $H$. Since the sequence $\left(y^{1 / n}\right)$ is bounded in norm, it will therefore suffice to show that $y^{1 / n} \xi \rightarrow p \xi$ for such $\xi$. Thus let $\epsilon>0$ and suppose $E_{y}(0, \epsilon) \xi=0$; also write $p^{\prime}=E_{y}[\epsilon, \infty)$ and $q=1-p=E_{y}(\{0\})$, and observe that $\xi=\left(p^{\prime}+q\right) \xi$ and $y^{1 / n} q=0$.

Now as $n$ goes to $\infty$, a functional calculus argument shows that $y^{1 / n} p^{\prime}$ converges to $p^{\prime}$ in operator norm. Thus

$$
y^{1 / n} \xi=y^{1 / n}\left(p^{\prime} \xi+q \xi\right)=y^{1 / n} p^{\prime} \xi \rightarrow p^{\prime} \xi=p \xi,
$$

as desired.

Lemma 8. $\Phi\left(x^{n}\right)^{1 / n} \leq x_{+}$for all $n \in \mathbf{N}$.

Proof. Since $x \preceq x_{+}$it follows from Olson's characterization of $\preceq$ that $x^{n} \leq x_{+}^{n}$. Thus $\Phi\left(x^{n}\right) \leq x_{+}^{n}$. By ([12, Proposition I.1.6]), taking $n$th roots preserves the inequality; thus $\Phi\left(x^{n}\right)^{1 / n} \leq x_{+}$.

Theorem 9. Let $\mathcal{N} \subset \mathcal{M}$ be von Neumann algebras, let $\Phi: \mathcal{M} \rightarrow \mathcal{N}$ be a faithful conditional expectation, and let $x \in \mathcal{M}$ be positive. Then the sequence $\Phi\left(x^{n}\right)^{1 / n}$ 
converges in the strong operator topology to $x_{+}$, the spectral order majorant of $x$ in $\mathcal{N}$.

Proof. Let $x_{n}=\Phi\left(x^{n}\right)^{1 / n}$; we want to show that $x_{+}-x_{n} \rightarrow 0$. By Lemma 8 , the elements $x_{+}-x_{n}$ are all positive; hence strong operator convergence to zero is equivalent to weak operator convergence to zero. Thus, we must show that $x_{+}-x_{n} \rightarrow 0$ (weak operator). By weak operator compactness of the unit ball, it will suffice to show that no weak operator cluster point of the sequence $\left(x_{+}-x_{n}\right)$ is strictly positive. Equivalently, we must show that no cluster point of $\left(x_{n}\right)$ is strictly less than $x_{+}$. By Theorem 6 , it will therefore suffice to show that $a E_{x_{+}}(a, \infty) \leq z$ for any weak operator cluster point $z$ of $\left(x_{n}\right)$.

Let $z$ be a weak operator cluster point of the sequence $\left(x_{n}\right)$. For $a \in \mathbf{R}$ define

$$
p=E_{x}(a, \infty) \quad \text { and } \quad q=E_{x_{+}}(a, \infty) .
$$

Now $x^{n} \geq a^{n} p$, hence $\Phi\left(x^{n}\right) \geq a^{n} \Phi(p)$, and taking $n$th roots as in Lemma 8 then yields $x_{n} \geq a \Phi(p)^{1 / n}$.

Let $p^{\prime}$ be the range projection of $\Phi(p)$. We claim that $p^{\prime} \geq p$. For otherwise,

$$
\left(1-p^{\prime}\right) p\left(1-p^{\prime}\right)>0
$$

hence

$$
\Phi\left(\left(1-p^{\prime}\right) p\left(1-p^{\prime}\right)\right)>0
$$

by faithfulness of $\Phi$, hence

$$
\left(1-p^{\prime}\right) \Phi(p)\left(1-p^{\prime}\right)>0
$$

a contradiction. (We are indebted to Lawrence Brown for this observation.) It then follows from the definition of $x_{+}$that $p^{\prime} \geq q$. Lemma 7 now implies that $a \Phi(p)^{1 / n} \rightarrow a p^{\prime} \geq a q$ (strong operator). Combining with the result of the last paragraph then shows that $z \geq a q$, as desired.

Corollary 10. Let $x$ and $y$ be positive operators on a Hilbert space. Then the operators $\left(x^{n}+y^{n}\right)^{1 / n}$ converge in the strong operator topology to the spectral order join of $x$ and $y$.

Proof. Let $\mathcal{M}=B(H) \oplus B(H)$ and $\mathcal{N}=B(H)$, with $\mathcal{N}$ embedded diagonally in $\mathcal{M}$ via $z \mapsto(z, z)$. Then the map $\Phi:\left(z, z^{\prime}\right) \mapsto\left(z+z^{\prime}\right) / 2$ is a faithful conditional expectation from $\mathcal{M}$ onto $\mathcal{N}$. According to Theorem 9, then, for any positive element $x \oplus y \in \mathcal{M}$

$$
\Phi\left((x \oplus y)^{n}\right)^{1 / n}=\Phi\left(x^{n} \oplus y^{n}\right)^{1 / n} \rightarrow z
$$

where $z$ is the least element of $\mathcal{N}$, relative to the spectral order, which dominates $x \oplus y$. Thus, $z$ is just the spectral order join of $x$ and $y$ and we have that

$$
\left(\frac{x^{n}+y^{n}}{2}\right)^{1 / n} \rightarrow z
$$

(strong operator). Since $(1 / 2)^{1 / n} \rightarrow 1$, this implies that $\left(x^{n}+y^{n}\right)^{1 / n} \rightarrow z$ as well.

We note that it is possible to prove Theorem 9 using Corollary 3 or Corollary 4 rather than Theorem 6 . This can be done by means of the following device. Choose $\epsilon>0$ and define $f: \mathbf{R} \rightarrow \mathbf{R}$ by

$$
f(t)=k \epsilon \quad \text { for } \quad k \in \mathbf{Z} \quad \text { and } \quad k \epsilon<t \leq(k+1) \epsilon ;
$$


then by functional calculus define

$$
x^{\prime}=f(x), \quad x_{n}^{\prime}=\Phi\left(\left(x^{\prime}\right)^{n}\right)^{1 / n}, \quad x_{+}^{\prime}=f\left(x_{+}\right) .
$$

Then $x_{+}^{\prime}$ is the spectral order majorant of $x^{\prime}$ in $\mathcal{N}$. Also $x^{n} \geq\left(x^{\prime}\right)^{n}$, hence $x_{n} \geq x_{n}^{\prime}$. In this way the problem is reduced to showing that $x_{n}^{\prime} \rightarrow x_{+}^{\prime}$ weak operator, which can be proved in the same way that we showed $x_{n} \rightarrow x_{+}$, but now requiring only Corollary 3 or Corollary 4 since $x_{+}^{\prime}$ has finite spectrum.

\section{REFERENCES}

1. T. Ando, Majorization, doubly stochastic matrices, and comparison of eigenvalues, Lin. Alg. Appl. 118 (1989), 163-248. MR 90g:15034

2. W. Arveson, On groups of automorphisms of operator algebras, J. Funct. Anal. 15 (1974), 217-243. MR 50:1016

3. L. G. Brown, Semicontinuity and multipliers of $C^{*}$-algebras, Can. J. Math. 40 (1988), 865988. MR 90a: 46148

4. J. Dixmier, Formes Linéaires sur un anneau d'opérateurs, Bull. de la Soc. Math. de France (1953), 9-39. MR 15:539a

5. R. V. Kadison, Order properties of bounded self-adjoint operators, Proc. Amer. Math. Soc. 2 (1951), 505-510. MR 13:47c

6. R. V. Kadison and J. R. Ringrose, Fundamentals of the Theory of Operator Algebras, Volume II, Academic Press (1986). MR 88d:46106

7. T. Kato, Spectral order and a matrix limit theorem, Linear and Multilinear Algebra 8 (1979), 15-19. MR 80j:47030

8. A. Lambert, personal communication.

9. M. P. Olson, The selfadjoint operators of a von Neumann algebra form a conditionally complete lattice, Proc. Amer. Math. Soc. 28 (1971), 537-544. MR 43:2528

10. G. K. Pedersen, Power ordering of positive operators, Kobenhavns Universitet Matematisk Institut Preprint Series \# 14 (1989).

11. S. Sherman, Order in operator algebras, Amer. J. Math. 73 (1951)), 227-232. MR 13:47d

12. M. Takesaki, Theory of Operator Algebras I, Springer-Verlag, 1979. MR 81e:46038

13. J. Tomiyama, On the projection of norm one in $W^{*}$-algebras, Proc. Japan Acad. 33 (1957), 608-612. MR 20:2635

14. M. Uchiyama, Powers and commutativity of selfadjoint operators, Math. Ann. 300 (1994), 643-647. MR 96a:47059

Department of Mathematics, University of California, Santa Barbara, California 93106

E-mail address: akemann@math.ucsb.edu

E-mail address: weaver@math.ucsb.edu 